\title{
TAKOTSUBO SYNDROME INDUCED BY MALIGNANT PHEOCHROMOCYTOMA IN A PATIENT WITH TYPE 2 PAPILLARY RENAL CELL CARCINOMA - A CASE REPORT
}

\author{
ANCA DIANA DEMEA, DAN-GRIGORE DUNCA, ROXANA ADINA RADU, \\ LUCIA AGOŞTON-COLDEA
}

Iuliu Hatieganu University of Medicine and Pharmacy, Cluj-Napoca, Romania

\begin{abstract}
The clinical presentation of the Takotsubo syndrome mimics an acute coronary syndrome with chest pain, ischemia-like ECG changes, mild to moderate myocardial enzyme elevation, and apical ballooning on echocardiography and ventriculography. On coronary angiography, epicardial coronary arteries are either normal or exhibit minimal atherosclerotic changes. Primary Takotsubo syndrome usually occurs in postmenopausal women in whom symptoms are triggered by emotional or physical stress, associated with catecholamine surges. Secondary Takotsubo syndrome may have multiple causes, including an increased catecholamine release due to pheochromocytoma.

We present the case of a 56-years-old woman with confirmed Takotsubo syndrome who was later diagnosed with pheochromocytoma and type 2 papillary renal cell carcinoma.
\end{abstract}

Keywords: Takotsubo syndrome, malignant pheochromocytoma, papillary renal cell carcinoma

\section{Introduction}

The clinical presentation of the Takotsubo syndrome mimics an acute coronary syndrome with chest pain, ST-segment elevation, T wave inversion, and apical ballooning on echocardiography and ventriculography. Invariably, the coronary arteries are found to be normal or exhibiting minimal atherosclerotic changes. Patients are often postmenopausal women in whom symptoms are triggered by emotional or physical stress, associated with catecholamine surges. Secondary Takotsubo syndrome symptoms may be triggered, however, by various factors, including an increased catecholamine release due to pheochromocytoma [1]. Although pheochromocytoma can occur as an isolated condition, associations to other neoplasia are not uncommon.

In the current paper, we present the case of a middleaged female patient with associated Takotsubo syndrome, pheochromocytoma and renal cell carcinoma.

\section{Case report}

A 52-year-old female with a history of arterial hypertension and type 2 diabetes mellitus presented to the

Manuscript received: 30.10.2017

Received in revised form: 16.12.2017

Accepted: 01.01.2018

Address for correspondence: ancademea@yahoo.com emergency room within 15 minutes from the sudden onset of constrictive chest pain, accompanied by dyspnea and diaphoresis. The symptoms occurred while the patient was attending a family wedding. Clinical examination revealed rhythmic cardiac sounds, with a rate of 80 beats/minute, blood pressure (BP) of 130/80 $\mathrm{mmHg}$, on both arms, and fine crackles in the lower half of both pulmonary fields. The 12-lead electrocardiogram was immediately recorded, showing ST-segment elevation in V1-V6, lead I and aVL, without mirror image (Figure 1). An echocardiography was performed in the emergency service, revealing wall-motion abnormalities of the left ventricle - severe mid-ventricular hypokinesia, apical ballooning and basal hypercontraction, with a left ventricle ejection fraction of $40 \%$ (Figure 2). Troponin I levels were $5.4 \mu \mathrm{g} / \mathrm{mL}$ (maximum normal value for the used kit: $<0.013 \mu \mathrm{g} / \mathrm{L}$ ). The case was interpreted as a Killip II antero-lateral acute myocardial infarction and coronary angiography was immediately performed, yielding normal epicardial coronary arteries. Hence, contrast was injected into the left ventricle, revealing apical ballooning and basal hypercontractility.

Considering the acute chest pain, ST segment elevations, and apical ballooning on echocardiography and ventriculography in the presence of normal coronary arteries, the case was interpreted as a Takotsubo syndrome, 
according to the criteria of the Heart Failure Association of the European Society of Cardiology [1].

Over the next few days, the patient had short episodes of atrial fibrillation, atrial tachycardia and multiple ventricular premature beats, which resolved under treatment with beta-blockers, as did ST segment elevations and LV contraction abnormalities. During hospitalization, higher BP values were recorded, ranging between 130 $170 \mathrm{mmHg}$ (systolic) and $70-90 \mathrm{mmHg}$ (diastolic). The patient was discharged after five days of hospitalization, on bisoprolol, atorvastatin, aspirin, furosemide, spironolactone, and insulin.

Three years later, the patient presented to the emergency room with high $\mathrm{BP}(180 / 78 \mathrm{mmHg})$ and diffuse muscle pain, elevated creatine kinase and creatinine levels, due to an underlying episode of rhabdomyolysis. She was hospitalized, statin was discontinued, and the antihypertensive treatment was re-adjusted by addition of an angiotensin-conversion enzyme inhibitor, but BP values could not be fully stabilized. The 24-hour BP monitoring showed diurnal values averaging 164/88 $\mathrm{mmHg}$, and a dipper profile, with mean nocturnal BP values of 140/72 mmHg.

The abdominal ultrasound revealed a left adrenal mass of 12.4/10.4/10.0 cm, neatly delineated and well vascularized, mostly transonic, with several hyperechoic areas, prompting further investigation by Computed Tomography (CT). The contrast-enhanced CT scan showed a heterogeneously enhancing left suprarenal tumor of $8.7 / 12.7 / 12.0 \mathrm{~cm}$, graded T4NxM0, with invasion into the left renal vein, left renal vein thrombosis and a left renal tumor, graded T3NxM0 (Figure 3).

Considering the location of the tumor and the history of high BP and apical ballooning syndrome, pheochromocytoma was suspected. Treatment with betablockers and angiotensin-conversion enzyme inhibitors was ceased and replaced by calcium channel blockers for two weeks. Then, metanephrines were determined in 24hour urine, yielding increased levels $-38.7 \mathrm{mg} / 24 \mathrm{~h}$ (normal range $0-1 \mathrm{mg} / 24 \mathrm{~h}$ ), and, thus, confirming the diagnosis of pheochromocytoma [2]. The patient was prescribed an alpha-blocker and referred for surgery.

Typical extirpative surgery was performed: left suprarenalectomy, perifascial nephrectomy and cavotomy with tumoral thrombectomy. The histopathology report confirmed malignant pheochromocytoma and type 2 papillary renal cell carcinoma. There were no perioperative complications and the patient recovered well after surgery. BP was stabilized and anti-hypertensive treatment was no longer required.

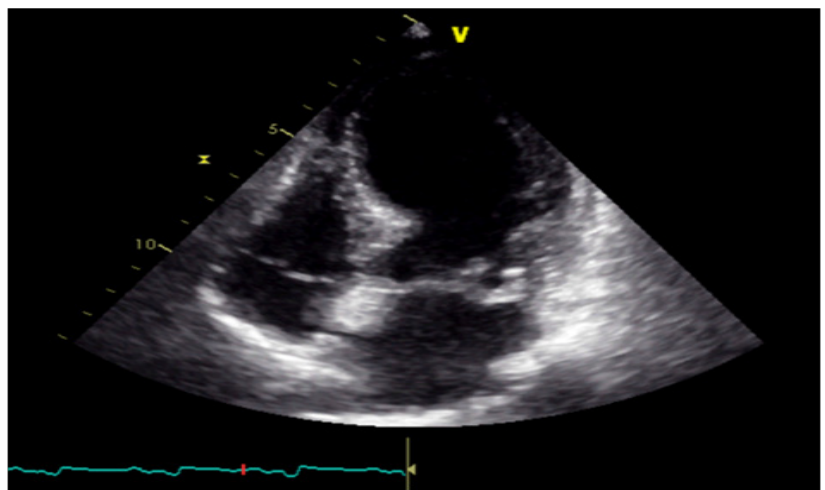

Figure 2. Echocardiogram - apical 4 chamber view showing apical ballooning.

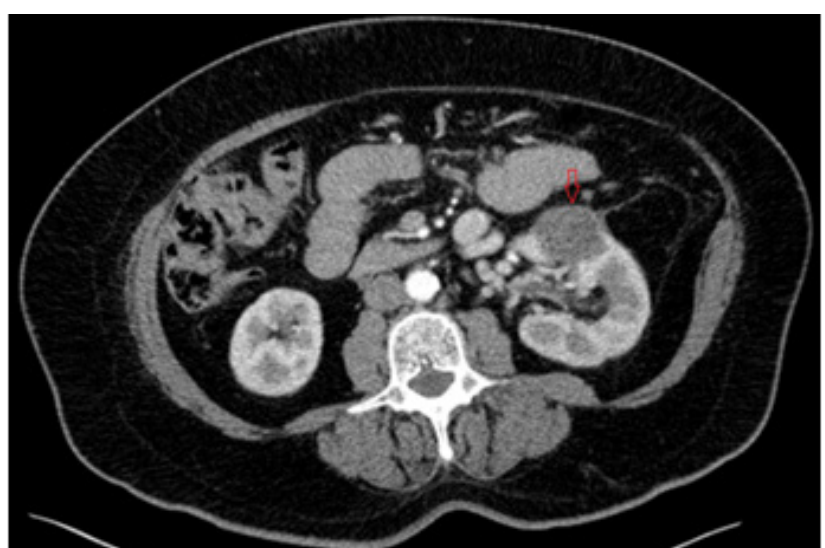

Figure 1. 12-lead electrocardiogram - sinus rhythm, heart rate 72 beats minute, QRS axis $+15^{\circ}$, ST segment elevation in V1-V6, D I and aVL.

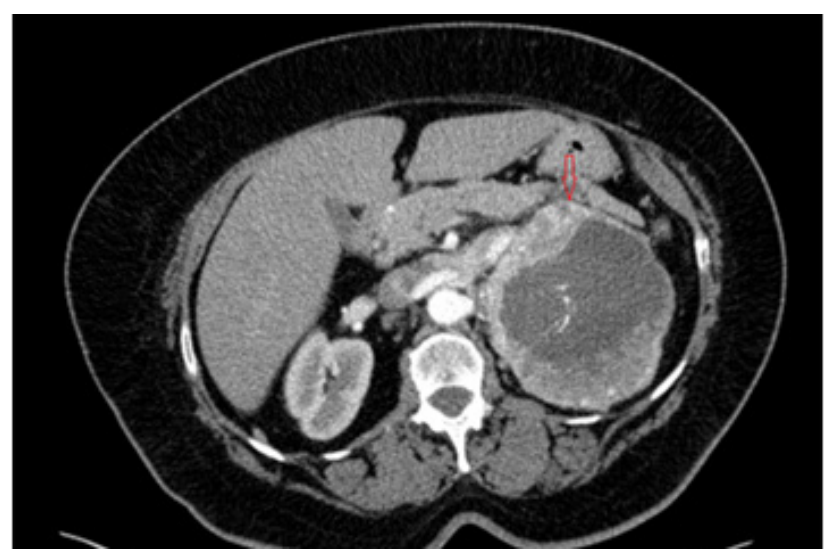

Figure 3. Contrast-enhanced CT - Figure 3A. Axial view: suprarenal, irregularly shaped tumor, 8.7/12.7/12.0 cm, with heterogeneous aspect, central necrosis, and infracentimetric calcifications; the mass invades the left renal vena cava by a tumoral thrombus, $7.3 \mathrm{~cm}$ long, extending to the origin of the inferior vena cava, and displaces the splenic vein anteriorly, without impeding the flow. Collateral circulation is visible around the mass. Figure $3 \mathrm{~B}$. Axial view: The left kidney is enlarged due to two simple cortical cysts (inferior pole) and a medio-renal natively hypodense lesion of $2.7 / 4.2 / 3.7$ cm, with heterogeneous contrast enhancement, suggesting a Bosniak III renal cyst. 


\section{Discussion}

The reported case illustrates the progression of a malignant pheochromocytoma causing rhabdomyolysis and acute renal failure, associated with renal cell carcinoma, in a diabetic patient with previously diagnosed Takotsubo syndrome.

Although pheochromocytoma is an acknowledged trigger of secondary Takotsubo syndrome [1-3], the association with renal cell carcinoma is a rare occurrence that should raise suspicion of a familial syndrome. Although the association between pheochromocytoma and renal cell carcinoma was reported as early as 1998 [4], the genetic background for this condition has only recently been investigated [5].

Pheochromocytoma and renal cell carcinoma can occur as a part of complex syndromes, such as type 2 multiple endocrine neoplasia (as a consequence of RET proto-oncogene mutations), Von Hippel-Lindau disease (mutations of the VHL gene), or pheochromocytomaparaganglioma syndromes (heterozygous germline mutations of succinate dehydrogenase subunits $\mathrm{B}, \mathrm{C}$, and D). In fact, up to 15 susceptible genes have been identified so far, including the recently reported FH and TMEM127 germline mutations, which regulate kinase receptor signaling pathways [5].

\section{Conclusion}

Pheochromocytoma can induce secondary Takotsubo syndrome and the diagnosis should be pursued especially when treatment-resistant arterial hypertension occurs. Although the association of pheochromocytoma and renal cell carcinoma is rare, in confirmed cases family screening should be considered.

\section{References}

1. Lyon AR, Bossone E, Schneider B, Sechtem U, Citro R, Underwood SR, et al. Current state of knowledge on Takotsubo syndrome: a Position Statement from the Taskforce on Takotsubo Syndrome of the Heart Failure Association of the European Society of Cardiology. Eur J Heart Fail. 2016;18(1):8-27.

2. Sharkey SW, McAllister N, Dassenko D, Lin D, Han K, Maron BJ. Evidence That High Catecholamine Levels Produced by Pheochromocytoma May be Responsible for Tako-Tsubo Cardiomyopathy. Am J Cardiol. 2015;115(11):1615-1618.

3. Y-Hassan S. Clinical Features and Outcome of PheochromocytomaInduced Takotsubo Syndrome: Analysis of 80 Published Cases. Am J Cardiol. 2016;117(11):1836-1844.

4. Ohgaki K, Kondo Y, Kimura G, Senga Y, Miura T, Hasegawa J, et al. Adrenal pheochromocytoma associated with renal cell carcinoma: report of two cases. Hinyokika Kiyo. 1998;44(3):167-170.

5. Hernandez KG, Ezzat S, Morel CF, Swallow C, Otremba M, Dickson BC, et al. Familial pheochromocytoma and renal cell carcinoma syndrome: TMEM127 as a novel candidate gene for the association. Virchows Arch. 2015;466(6):727-732. 